\title{
On the accuracy of the perturbative approach for strong lensing: local distortion for pseudo-elliptical models
}

\author{
Habib S. Dúmet-Montoya, ${ }^{1,2 \star}$ Gabriel B. Caminha, ${ }^{1,2 \star}$ Bruno Moraes, ${ }^{1,2}$ \\ Martin Makler, ${ }^{1,2}$ Mandeep S. S. Gill ${ }^{3,4}$ and Basílio X. Santiago ${ }^{2,5}$ \\ ${ }^{1}$ Instituto de Cosmologia, Relatividade e Astrofísica - ICRA, Centro Brasileiro de Pesquisas Físicas, Rua Dr. Xavier Sigaud 150, CEP 22290-180, Rio de \\ Janeiro, RJ, Brazil \\ ${ }^{2}$ Laboratório Interinstitucional de e-Astronomia - LIneA, Rua Gal. José Cristino 77, CEP 20921-400, Rio de Janeiro, RJ, Brazil \\ ${ }^{3}$ Kavli Institute for Particle Astrophysics \& Cosmology, SLAC National Accelerator Laboratory, 2575 Sand Hill Road, Menlo Park, CA 94025, USA \\ ${ }^{4}$ Center for Cosmology and Astro-Particle Physics, Department of Physics, The Ohio State University, 191 West Woodruff Avenue, Columbus, OH 43210, USA \\ ${ }^{5}$ Departamento de Astronomia, Universidade Federal do Rio Grande do Sul, Av. Bento Gonçalves 9500, Porto Alegre, RS 91501-970, Brazil
}

Accepted 2013 May 27. Received 2013 May 10; in original form 2012 December 31

\begin{abstract}
The perturbative approach (PA) introduced by Alard provides analytic solutions for gravitational arcs by solving the lens equation linearized around the Einstein ring solution. This is a powerful method for lens inversion and simulations in which it can be used, in principle, for generic lens models. In this paper, we aim to quantify the domain of validity of this method for three quantities derived from the linearized mapping: caustics, critical curves and the deformation cross-section (i.e. the arc cross-section in the infinitesimal circular source approximation). We consider lens models with elliptical potentials, in particular the singular isothermal elliptic potential and pseudo-elliptical Navarro-Frenk-White models. We show that the PA is exact for this first model. For the second, we obtain constraints on the model parameter space (given by the potential ellipticity parameter $\varepsilon$ and characteristic convergence $\kappa_{s}$ ) such that the PA is accurate for the aforementioned quantities. In this process, we obtain analytic expressions for several lensing functions, which are valid for the PA in general. The determination of this domain of validity could have significant implications for the use of the PA, but it still needs to be probed with extended sources.
\end{abstract}

Key words: gravitational lensing: strong-galaxies: clusters: general-dark matter.

\section{INTRODUCTION}

Gravitational arc systems can be used as a powerful probe of the matter distribution of galaxies and galaxy clusters acting as lenses (Kovner 1989; Miralda-Escudé 1993a; Hattori, Watanabe \& Yamashita 1997). Further, their abundance can be used to constrain cosmological models (Bartelmann et al. 1998, 2003; Oguri, Taruya \& Suto 2001; Golse, Kneib \& Soucail 2002). This motivated several arc searches to be carried out, both in wide field surveys (Gladders et al. 2003; Cabanac et al. 2007; Estrada et al. 2007; Belokurov et al. 2009; Kneib et al. 2010; Kubo et al. 2010; Gilbank et al. 2011; Wen, Han \& Jiang 2011; Bayliss 2012; More et al. 2012; Wiesner et al. 2012; Erben et al., in preparation), as well as in images targeting know clusters (Luppino et al. 1999; Zaritsky \& Gonzalez 2003; Sand et al. 2005; Smith et al. 2005; Hennawi et al. 2008; Horesh et al. 2010; Kausch et al. 2010; Furlanetto et al. 2013a). Upcom- ing wide field imaging surveys, such as the Dark Energy Survey ${ }^{1}$ (Abbott et al. 2005; Annis et al. 2005), which started operations in 2012, are expected to detect of the order of $10^{3}$ strong lensing systems, about an order of magnitude increase with respect to the current largest surveys.

Two primary approaches have been followed in practical applications of gravitational arc systems. On the one hand, inverse modelling attempts to 'deproject' the arcs in individual lens systems to determine lens and source properties (Kneib et al. 1993; Keeton 2001b; Golse et al. 2002; Comerford et al. 2006; Wayth \& Webster 2006; Jullo et al. 2007, 2010). On the other hand, arc statistics (Wu \& Hammer 1993; Bartelmann \& Weiss 1994; Grossman \& Saha 1994) aims at counting the number of arcs in cluster samples and comparing with the predictions from cosmological models.

Both approaches require the lens equation to be solved numerically for finite sources numerous times. The inverse modelling typically needs arc images obtained from a multidimensional space 
of source positions and lens parameters scanned during the minimization process to find the best solution for sources and lenses (e.g., Wayth \& Webster 2006). For this reason, analyses using the inverse modelling are often restricted to simple lens models, in particular models with elliptic lens potentials (so-called pseudoelliptical) and/or to point sources, for example, considering bright spots in arcs as multiple images of point sources (Keeton 2001b; Wayth \& Webster 2006; Jullo et al. 2007; Oguri 2010).

For arc statistics, predictions for the arc cross-section must be derived as a function of source and lens properties, and the cosmological model again by obtaining a large set of arc images (Miralda-Escudé 1993b; Bartelmann \& Weiss 1994; Meneghetti et al. 2001; Meneghetti, Bartelmann \& Moscardini 2003; Oguri, Lee \& Suto 2003). The cross-section is then convolved with the distribution of lens properties expected in a given cosmology and convolved with the source distribution. Another approach is to use directly high-resolution $\mathrm{N}$-body simulations obtaining arc images by ray tracing through the mass distribution for a large number of sources (Meneghetti et al. 2008; Horesh et al. 2011; Boldrin et al. 2012).

It is therefore useful to develop approximate methods for obtaining gravitational arcs, which will be particularly useful given the increase of strong lensing systems to be discovered by the next generation wide-field surveys. A most promising technique for this purpose is given by the perturbative approach (PA; Alard 2007, 2008), which provides an approximate solution for the lens equation close to the Einstein ring, leading to analytic solutions for arcs.

The power of this approach is that it can be applied, in principle, to generic lens models, including those arising from simulations. The method is suitable for large tangential arcs, since the solutions are accurate for images located close to the Einstein ring corresponding to the circularly averaged lensing potential.

Another important feature of the method is that it naturally reproduces arcs resulting from the merger of multiple images, which cannot be accounted for with other approximate methods for arcs proposed in the literature (e.g., Keeton 2001a; Fedeli et al. 2006). Such merger arcs are key for lens inversion methods and also play an important role in the arc cross-section (Rozo et al. 2008).

The PA has already been used for inverse modelling in Alard (2009, 2010). Given that it reproduces arc contours that can be associated with isophotes, it could also be used to simulate the brightness distribution of arcs, in a similar way to what was implemented in Furlanetto et al. (2013b) for arc shaped contours.

An important issue for practical applications of this approach is the determination of its domain of validity. This topic is discussed in Alard (2007), comparisons with arc simulations are presented in Peirani et al. (2008), and a recent work by Habara \& Yamamoto (2011) has investigated arcs in several configurations for a pseudoelliptical model in this approach. However, a systematic study of its limit of applicability has not yet been carried out. In this paper, we make a first attempt to determine a domain of validity of the method in terms of the parameter space of the lens model. We will restrict to the simple case of pseudo-elliptical models, which are nevertheless widely used for the inverse modelling. Moreover, for simplicity, we will restrict the comparisons with the exact solution for three quantities connected to arcs, but which do not involve the lensing of finite sources. We expect that the limits obtained here can be connected to the domain of validity for arcs and extended for more general models, but this is left for subsequent explorations.

In this work, our purpose is twofold. The first is to explore the application of the PA to determine quantities arising from the local lens mapping, such as the arc cross-section for infinitesimal circular sources (deformation cross-section). The second is to determine a domain of validity such that the critical curves, caustics and deformation cross-section are accurately obtained. This study is performed for the pseudo-elliptical Navarro-Frenk-White (PNFW) model, determining regions of its parameter space where the PA provides a good approximation for these quantities. We also consider the singular isothermal elliptic potential (SIEP) model and show that the solution of the PA is exact in this case.

The outline of this paper is as follows: in Section 2, we present a few basic results of gravitational lensing theory, introduce the radial lens models to be used in this work and discuss models with elliptic lensing potentials. In Section 3, we review the PA, present its application to the computation of the deformation cross-section and discuss its implementation to pseudo-elliptical models. In Section 4, we establish a metric for the comparison between the PA and the exact solution for critical curves and caustics, and determine a domain of validity for the PA. In Section 5, we summarize the results and present concluding remarks.

\section{BASICS OF GRAVITATIONAL LENSING: DEFINITIONS AND NOTATION}

In this section, we present a brief review of the lensing theory to set up the notation and to define the quantities associated with pseudoelliptical models. For a more detailed description see, e.g., chapter 8 of Schneider, Elhers \& Falco (1992), chapter 6 of Petters, Levine \& Wambsganss (2001) and chapter 3 of Mollerach \& Roulet (2002).

The lens equation relates the two-dimensional position (with respect to the optical axis) of the observed images $\xi$ to those of the sources $\eta$. We may choose a length-scale $\xi_{0}$ and define $\boldsymbol{x}=$ $\boldsymbol{\xi} / \xi_{0}$ and $\boldsymbol{y}=\boldsymbol{\eta} / \eta_{0}$, with $\eta_{0} \equiv \frac{D_{\mathrm{OS}}}{D_{\mathrm{OL}}} \xi_{0}$, where $D_{\mathrm{OL}}$ and $D_{\mathrm{OS}}$ are the angular diameter distances from the observer to the lens and source, respectively. Using these definitions the lens equation is written as

$\boldsymbol{y}=\boldsymbol{x}-\boldsymbol{\alpha}(\boldsymbol{x})=\boldsymbol{x}-\nabla_{x} \varphi(\boldsymbol{x})$,

where $\boldsymbol{\alpha}(\boldsymbol{x})$ is the 'dimensionless' deflection angle and $\varphi(\boldsymbol{x})$ is the 'dimensionless' lensing potential.

The local distortion in the lens plane is described by the Jacobian matrix of equation (1)

$\mathbf{J}=\left(\frac{\partial \boldsymbol{y}}{\partial \boldsymbol{x}}\right)_{i j}=\delta_{i j}-\partial_{i} \alpha_{j}(\boldsymbol{x})$.

The two eigenvalues of this matrix are written as $\lambda_{\mathrm{r}}=1-\kappa+\gamma$ and $\lambda_{\mathrm{t}}=1-\kappa-\gamma$, where $\kappa$ and $\gamma$ are the convergence and the shear given below. Points satisfying the conditions $\lambda_{\mathrm{r}, \mathrm{t}}=0$ define the radial and tangential critical curves, respectively. Mapping these curves onto the source plane, we obtain the caustics.

For axially symmetric models, the deflection angle, convergence and shear are given by

$$
\begin{aligned}
& \alpha(x)=\frac{\mathrm{d} \varphi_{0}(x)}{\mathrm{d} x}=x \frac{\bar{\Sigma}\left(\xi_{0} x\right)}{\Sigma_{\text {crit }}}, \\
& \kappa(x)=\frac{1}{2}\left[\frac{\alpha(x)}{x}+\frac{\mathrm{d} \alpha(x)}{\mathrm{d} x}\right], \\
& \gamma(x)=\frac{1}{2}\left[\frac{\alpha(x)}{x}-\frac{\mathrm{d} \alpha(x)}{\mathrm{d} x}\right],
\end{aligned}
$$

where $\bar{\Sigma}\left(\xi_{0} x\right)$ is the mean surface density within a radius $x$ and $\Sigma_{\text {crit }}$ is the critical surface density. 
In this work, one model we will make use of is the singular isothermal sphere (SIS), which is useful to model lenses at the galactic scale. Its dimensionless lensing potential, deflection angle, convergence and shear are given by (Turner, Ostriker \& Gott 1984; Schneider et al. 1992; van de Ven, Mandelbaum \& Keeton 2009)

$\varphi_{0}(x)=x, \quad \alpha(x)=1, \quad \kappa(x)=\gamma(x)=\frac{1}{2}$,

where we choose the Einstein radius to be the characteristic scale

$\xi_{0}=R_{\mathrm{E}}=\frac{\sigma_{v}^{2}}{G \Sigma_{\text {crit }}}$,

where $\sigma_{v}$ is the velocity dispersion. From this potential, analytic solutions of the lens equation can be obtained for finite sources (Dobler \& Keeton 2006; Dúmet-Montoya 2011).

We will also make use of the Navarro-Frenk-White (NFW) model (Navarro, Frenk \& White 1996, 1997), often used to represent lenses in the galaxy-to-galaxy cluster mass scales. This model has two independent parameters $r_{\mathrm{s}}$ and $\rho_{s}$. By fixing $\xi_{0}=r_{\mathrm{s}}$ and defining the characteristic convergence as

$\kappa_{s}=\frac{\rho_{s} r_{\mathrm{s}}}{\Sigma_{\mathrm{crit}}}$,

the lensing potential is given by (Bartelmann 1996)

$\varphi_{0}(x)=4 \kappa_{s}\left(\frac{1}{2} \log ^{2} \frac{x}{2}-2 \operatorname{arctanh}^{2} \sqrt{\frac{1-x}{1+x}}\right)$,

which is a function of the parameter $\kappa_{s}$ alone.

Models with elliptical potentials (the so-called pseudo-elliptical models) provide simple analytical solutions for some lensing quantities (Blandford \& Kochanek 1987; Kassiola \& Kovner 1993; Kneib 2001). They have been widely used in lens inversion problems and are implemented in several public codes for lens inversion such as GRAVLENS (Keeton 2001b), LENSVIEW (Wayth \& Webster 2006), LENSTOOL (Jullo et al. 2007) and GLAFIC (Oguri 2010). They have also been used for arc simulations (Oguri 2002; Meneghetti et al. 2003, 2007).

Pseudo-elliptical models, with potential $\varphi_{\varepsilon}(\boldsymbol{x})$, are built from a given axially symmetric potential $\varphi_{0}(x)$ by replacing the radial coordinate $x$ by

$\tilde{x}=\sqrt{a_{1} x_{1}^{2}+a_{2} x_{2}^{2}}=x \Delta_{\phi}$,

where

$$
\Delta_{\phi} \equiv \sqrt{a_{1} \cos ^{2} \phi+a_{2} \sin ^{2} \phi},
$$

such that the ellipticity of the lensing potential is

$\varepsilon_{\varphi}=1-\sqrt{\frac{a_{1}}{a_{2}}}$,

where the orientation was chosen such that the major axis of the ellipse is along the $x_{1}$ axis (i.e. $a_{2}>a_{1}$ ). The deflection angle, convergence and shear can be written as combinations of the lensing functions of the corresponding axially symmetric model for any choice of $a_{1}$ and $a_{2}$ (Dúmet-Montoya, Caminha \& Makler 2012).

The SIEP and PNFW models are obtained by following this procedure for the potentials given in equations (6) and (8), respectively.

\section{PERTURBATIVE APPROACH}

For a given lens model, the PA allows one to obtain analytic solutions for arcs as perturbations of the Einstein ring solution. In this work, we investigate the limits of applicability of the PA, by considering simple non-axially symmetric models and by looking at local properties of the lens mapping, instead of lensed finite sources.

In this section, we briefly review the PA and use it for the derivation of the caustics and critical curves, the deformation cross-section and quantities needed for its computation. The method is also applied to models with elliptical lensing potentials.

\subsection{Lens equation}

The gist of the PA for gravitational arcs developed by Alard (2007, 2008) is to obtain an analytic solution for the lens equation considering the lens as a perturbation of an axially symmetric configuration and the source position as a small deviation from the optical axis (i.e. positioned transversely away from perfect observer-lens-source alignment). In other words, the arcs are found as perturbations of the Einstein ring configuration. In this work, we will consider the thin lens and the single lens plane approximations, which imply a unique solution for the Einstein ring (Werner, An \& Evans 2008).

The Einstein ring is the image of a source aligned with an axially symmetric lens (with lensing potential $\varphi_{0}$ ). Its radius $x_{\mathrm{E}}$ is obtained by solving $\lambda_{t}(\boldsymbol{x})=0$ at the centre of the source plane, i.e.

$x-\frac{\mathrm{d} \varphi_{0}}{\mathrm{~d} x}=0$.

Arcs can be obtained by perturbing the equation above either by shifting the position of the source away from the optical axis and/or by adding a non-circular perturbation to the lensing potential. These perturbations are described by

$\boldsymbol{y}=\delta y, \varphi(\boldsymbol{x})=\varphi_{0}(x)+\delta \psi(\boldsymbol{x})$.

These perturbations are assumed to be of the same order in $\epsilon$ (the strength of the perturbation) throughout the following calculations, such that

$\delta y=\epsilon \bar{y}, \quad \delta \psi(\boldsymbol{x})=\epsilon \bar{\varphi}(\boldsymbol{x})$.

The response to such perturbations is given by the displacement of the radial coordinate in the lens plane, ${ }^{2}$ i.e. $x=x_{\mathrm{E}} \rightarrow x=x_{\mathrm{E}}+\delta x$, where we also assume the same order in $\epsilon$ such that $\delta x=\epsilon \bar{x}$.

To find $\epsilon \bar{x}$, we solve equation (1) by expanding the solution around $x=x_{\mathrm{E}}$. Expanding the lensing potential in a Taylor series around $x=x_{\mathrm{E}}$, we have

$\varphi(\boldsymbol{x})=\sum_{n=0}^{\infty}\left[C_{n}+f_{n}(\phi)\right](\epsilon \bar{x})^{n}$,

where

$C_{n} \equiv \frac{1}{n !}\left[\frac{\mathrm{d}^{n} \varphi_{0}}{\mathrm{~d} x^{n}}\right]_{x=x_{\mathrm{E}}}$,

$f_{n}(\phi) \equiv \frac{1}{n !}\left[\frac{\partial^{n} \delta \psi}{\partial x^{n}}\right]_{x=x_{\mathrm{E}}}=\frac{\epsilon}{n !}\left[\frac{\partial^{n} \bar{\varphi}}{\partial x^{n}}\right]_{x=x_{\mathrm{E}}}$

\footnotetext{
${ }^{2}$ Note that in Alard (2007, 2008), $\xi_{0}=x_{\mathrm{E}}$ was used as a characteristic scale. This choice is equivalent to setting $x_{\mathrm{E}}=1$ in our equations. In this work, we have made the choice of keeping $x_{\mathrm{E}}$ explicitly in the equations for more generality, allowing us, for example, to choose another characteristic scale of the problem.
} 
Inserting $x=x_{\mathrm{E}}+\epsilon \bar{x}$ and (13) into equation (1), we find that the resulting equation at zeroth order in $\epsilon$ is

$x_{\mathrm{E}}=C_{1}$,

which is the Einstein ring equation. Using the relations above and $\delta x=\epsilon \bar{x}$, the resulting equation at the first order in $\epsilon$ is given by

$y_{1}=\left(\kappa_{2} \delta x-f_{1}\right) \cos \phi+\frac{1}{x_{\mathrm{E}}} \frac{\mathrm{d} f_{0}}{\mathrm{~d} \phi} \sin \phi$,

$y_{2}=\left(\kappa_{2} \delta x-f_{1}\right) \sin \phi-\frac{1}{x_{\mathrm{E}}} \frac{\mathrm{d} f_{0}}{\mathrm{~d} \phi} \cos \phi$,

where $\kappa_{2} \equiv 1-2 C_{2}$. From equations (3)-(5) we have

$\frac{\mathrm{d}^{2} \varphi_{0}(x)}{\mathrm{d} x^{2}}=2 \kappa(x)-\frac{\alpha(x)}{x}$,

and therefore $\kappa_{2}$ can be expressed as

$\kappa_{2}=2-2 \kappa\left(x_{\mathrm{E}}\right)$.

Equation (16) is the lens equation in the PA. It can be solved for $\delta x$ for each angular position $\phi$ of the source, given a perturbation described by $f_{n}(\phi)$. To obtain the images of a finite source, we must first parametrize its boundary. Then, by varying $\phi$ from 0 to $2 \pi$, each point of that boundary is mapped to the lens plane through equations (16). As a result, a new equation with separated radial and angular components is formed, whose solution is obtained straightforwardly (Alard 2007, 2008; Peirani et al. 2008; Dúmet-Montoya 2011).

It is important to emphasize that the solutions $\left(x_{i}, \phi_{i}\right)$ of equation (16) are valid only to first order in the perturbations in equation (12), i.e. only for points near the Einstein ring. For points far from this curve, the solutions are not expected to be highly accurate. For this reason, the PA is particularly useful for applications involving tangential arcs. In this work, instead of using finite sources, we focus on the potential applicability of this method to quantities based on the local mapping as a first step to quantify the differences with the exact solutions.

\subsection{Local mapping}

The Jacobian matrix for the lens mapping is

$\mathbf{J}=\left(\frac{\partial \boldsymbol{y}}{\partial \boldsymbol{x}}\right)_{i j}=\sum_{k}\left(\mathbf{J}_{\mathrm{S} \rightarrow \mathrm{L}, \mathrm{pol}}\right)_{i k}\left(\mathbf{J}_{\mathrm{L}, \mathrm{pol} \rightarrow \mathrm{cart}}\right)_{k j}$,

where $\mathbf{J}_{\mathrm{S} \rightarrow \mathrm{L} \text {, pol }}$ is the Jacobian of the transformation from the lens plane to the source plane in polar coordinates from equation (16) and $\mathbf{J}_{\mathrm{L}, \text { pol } \rightarrow \text { cart }}$ is the standard Jacobian matrix from polar to Cartesian coordinates. The calculation of the eigenvalues of the lens mapping is then straightforward from the equation above and they are given by

$\lambda_{\mathrm{t}}=-\frac{1}{x}\left[\frac{1}{x_{\mathrm{E}}} \frac{\mathrm{d}^{2} f_{0}}{\mathrm{~d} \phi^{2}}-\left(\kappa_{2} \delta x-f_{1}\right)\right]$,
$\lambda_{\mathrm{r}}=\kappa_{2}$.

Therefore, the radial coordinate of the tangential critical curve is

$x_{\mathrm{t}}(\phi)=x_{\mathrm{E}}+\delta x_{\mathrm{t}}(\phi)=x_{\mathrm{E}}+\frac{1}{\kappa_{2}}\left(f_{1}+\frac{1}{x_{\mathrm{E}}} \frac{\mathrm{d}^{2} f_{0}}{\mathrm{~d} \phi^{2}}\right)$,

and the parametric equations of the critical curve are simply

$x_{1 \mathrm{t}}=x_{\mathrm{t}}(\phi) \cos \phi$,

$x_{2 \mathrm{t}}=x_{\mathrm{t}}(\phi) \sin \phi$.
Inserting $\delta x_{\mathrm{t}}$ in equation (16), the parametric equations of the tangential caustic are found to be

$$
\begin{gathered}
y_{1 t}=\frac{1}{x_{\mathrm{E}}} \frac{\mathrm{d}^{2} f_{0}}{\mathrm{~d} \phi^{2}} \cos \phi+\frac{1}{x_{\mathrm{E}}} \frac{\mathrm{d} f_{0}}{\mathrm{~d} \phi} \sin \phi, \\
y_{2 t}=\frac{1}{x_{\mathrm{E}}} \frac{\mathrm{d}^{2} f_{0}}{\mathrm{~d} \phi^{2}} \sin \phi-\frac{1}{x_{\mathrm{E}}} \frac{\mathrm{d} f_{0}}{\mathrm{~d} \phi} \cos \phi .
\end{gathered}
$$

\subsection{Constant distortion curves}

For infinitesimal circular sources, the length-to-width ratio of arcs can be approximated by the ratio of the eigenvalues of the lens mapping Jacobian matrix (Wu \& Hammer 1993; Bartelmann \& Weiss 1994; Hamana \& Futamase 1997)

$\frac{L}{W} \simeq\left|R_{\lambda}(\boldsymbol{x})\right| \equiv\left|\frac{\lambda_{\mathrm{r}}(\boldsymbol{x})}{\lambda_{\mathrm{t}}(\boldsymbol{x})}\right|$.

Under this approximation, it is possible to define a region where gravitational arcs are expected to form by fixing a value for the threshold length-to-width ratio $R_{\mathrm{th}}$. Such region is limited by the curves $R_{\lambda}= \pm R_{\text {th }}$ (constant distortion curves). Although the condition (22) does not hold for merger arcs (Rozo et al. 2008), nor for large or elliptical sources, the curves defined above still provide a typical scale for the region of arc formation. In this work, we adopt the common choice $R_{\mathrm{th}}=10$ (unless explicitly stated otherwise). We denote the radial coordinates of these curves as $x_{\lambda}$. They are obtained by solving $R_{\lambda}(x)= \pm R_{\mathrm{th}}$, with $\lambda_{\mathrm{r}}$ and $\lambda_{\mathrm{t}}$ given in the PA by equation (19). It follows that

$x_{\lambda}(\phi)=x_{\mathrm{t}}(\phi) \times \begin{cases}\frac{R_{\mathrm{th}}}{R_{\mathrm{th}}-1}, & R_{\lambda}=+R_{\mathrm{th}}, \\ \frac{R_{\mathrm{th}}}{R_{\mathrm{th}}+1}, & R_{\lambda}=-R_{\mathrm{th}} .\end{cases}$

The constant distortion curves in the lens plane are therefore self-similar to the tangential critical curve. The mapping of these curves to the source plane is done by substituting $\delta x_{\lambda}=x_{\lambda}-x_{\mathrm{E}}$ in equation (16). For instance, the curve $R_{\lambda}=+R_{\mathrm{th}}$ has the following parametric equations:

$$
\begin{gathered}
y_{1 \lambda}=\frac{f_{1}+\kappa_{2} x_{\mathrm{E}}}{R_{\mathrm{th}}-1} \cos \phi+\frac{1}{x_{\mathrm{E}}}\left[\left(\frac{R_{\mathrm{th}}}{R_{\mathrm{th}}-1}\right) \frac{\mathrm{d}^{2} f_{0}}{\mathrm{~d} \phi} \cos \phi+\frac{\mathrm{d} f_{0}}{\mathrm{~d} \phi} \sin \phi\right], \\
y_{2 \lambda}=\frac{f_{1}+\kappa_{2} x_{\mathrm{E}}}{R_{\mathrm{th}}-1} \sin \phi+\frac{1}{x_{\mathrm{E}}}\left[\left(\frac{R_{\mathrm{th}}}{R_{\mathrm{th}}-1}\right) \frac{\mathrm{d}^{2} f_{0}}{\mathrm{~d} \phi} \sin \phi-\frac{\mathrm{d} f_{0}}{\mathrm{~d} \phi} \cos \phi\right] .
\end{gathered}
$$

The parametric equations of the $R_{\lambda}=-R_{\mathrm{th}}$ curve are given by the expressions above with the substitution $R_{\mathrm{th}}-1 \rightarrow R_{\mathrm{th}}+1$. There is no self-similarity between these curves and the tangential caustics.

\subsection{Deformation cross-section}

As mentioned in Section 1, the arc cross-section is a key ingredient in arc statistics calculations. It is defined as the effective area in the source plane such that sources within it will be mapped into images with $L / W \geq R_{\mathrm{th}}$. The definition of this area must take into account the image multiplicity given the source position (i.e. multiply imaged regions are counted multiple times; see, e.g., Meneghetti et al. 2003). The computation of the arc cross-section in general demands raytracing simulations, which are computationally expensive (MiraldaEscudé 1993b; Bartelmann \& Weiss 1994; Meneghetti et al. 2001, 2003; Oguri et al. 2003). An alternative is to use the infinitesimal circular source approximation, equation (22), which allows the 
computation of the arc cross-section to be carried out directly from the local mapping from lens to source plane. In this case, $\sigma_{R_{\mathrm{th}}}$ is computed in the lens plane by

$\sigma_{R_{\mathrm{th}}}=\eta_{0}^{2} \tilde{\sigma}_{R_{\mathrm{th}}}=\eta_{0}^{2} \int_{\left|R_{\lambda}\right| \geq R_{\mathrm{th}}} \frac{\mathrm{d}^{2} x}{|\mu(\boldsymbol{x})|}$

(see, e.g., Fedeli et al. 2006; Dúmet-Montoya et al. 2012; Caminha et al., in preparation), where $\mu=\left(\lambda_{\mathrm{r}} \lambda_{\mathrm{t}}\right)^{-1}$ is the magnification and the integral is performed over the region of arc formation above the chosen threshold. The quantity $\tilde{\sigma}_{R_{\mathrm{th}}}$ is known as the dimensionless deformation cross-section.

In the PA, the magnification can be written from equation (19) as

$$
|\mu(\boldsymbol{x})|^{-1}=\frac{\kappa_{2}^{2}}{x} \begin{cases}x_{\mathrm{t}}(\phi)-x, & x<x_{\mathrm{t}}(\phi), \\ x-x_{\mathrm{t}}(\phi), & x>x_{\mathrm{t}}(\phi),\end{cases}
$$

where $x_{\mathrm{t}}(\phi)$ is given in equation (20). Inserting the equation above in equation (25) and integrating the radial coordinate within the lower and upper limits given in equation (23), it is straightforward to obtain

$\tilde{\sigma}_{R_{\mathrm{th}}}=\kappa_{2}^{2} \frac{\left|R_{\mathrm{th}}\right|^{2}+1}{\left(\left|R_{\mathrm{th}}\right|^{2}-1\right)^{2}} \int_{0}^{2 \pi} x_{\mathrm{t}}^{2}(\phi) \mathrm{d} \phi$.

Note that $\tilde{\sigma}_{R_{\mathrm{th}}} \propto R_{\mathrm{th}}^{-2}$ for $R_{\mathrm{th}} \gg 1$, as expected from the behaviour of the deformation cross-section with $R_{\mathrm{th}}$ (Rozo et al. 2008; Caminha et al., in preparation).

For axially symmetric models $\left(x_{\mathrm{t}}=x_{\mathrm{E}}\right)$, the cross-section is given simply by

$\tilde{\sigma}_{R_{\mathrm{th}}}=2 \pi \kappa_{2}^{2} x_{\mathrm{E}}^{2} \frac{R_{\mathrm{th}}^{2}+1}{\left(R_{\mathrm{th}}^{2}-1\right)^{2}}$.

The expression above is exact for the SIS model (Bartelmann, Steinmetz \& Weiss 1995). For other axially symmetric models this expression is still an approximation, since the curves $R_{\lambda}= \pm R_{\text {th }}$ are obtained approximatively.

\subsection{Perturbative functions for pseudo-elliptical models}

We write the elliptical potential as

$\varphi_{\varepsilon}(\boldsymbol{x})=\varphi_{0}(x)+\left[\varphi_{0}(\tilde{x})-\varphi_{0}(x)\right]$,

such that the perturbed potential becomes

$\delta \psi(x, \phi)=\varphi_{0}(\tilde{x})-\varphi_{0}(x)$.

From the definitions (14) and using the identities (3)-(5), it follows that

$$
\begin{aligned}
f_{1}= & \frac{\tilde{x}_{\mathrm{E}}}{x_{\mathrm{E}}} \alpha\left(\tilde{x}_{\mathrm{E}}\right)-\alpha\left(x_{\mathrm{E}}\right), \\
\frac{\mathrm{d} f_{0}}{\mathrm{~d} \phi}= & \frac{x_{\mathrm{E}}^{2}}{2 \tilde{x}_{\mathrm{E}}} \alpha\left(\tilde{x}_{\mathrm{E}}\right)\left(a_{2}-a_{1}\right) \sin 2 \phi, \\
\frac{\mathrm{d}^{2} f_{0}}{\mathrm{~d} \phi^{2}}= & \frac{x_{\mathrm{E}}^{2}}{\tilde{x}_{\mathrm{E}}} \alpha\left(\tilde{x}_{\mathrm{E}}\right)\left(a_{2}-a_{1}\right) \cos 2 \phi \\
& -\frac{\gamma\left(\tilde{x}_{\mathrm{E}}\right)}{2}\left[\frac{x_{\mathrm{E}}^{2}}{\tilde{x}_{\mathrm{E}}}\left(a_{2}-a_{1}\right) \sin 2 \phi\right]^{2},
\end{aligned}
$$

where $\alpha$ and $\gamma$ are the deflection angle and shear of the corresponding axially symmetric lens. These expressions hold for any parametrization of the lensing potential ellipticity and for any pseudo-elliptical lens (Dúmet-Montoya et al. 2012).

For small values of the lensing potential ellipticity, equations (30) reduce to

$$
\begin{aligned}
f_{1} & =\frac{a_{1}-a_{2}}{2} \kappa\left(x_{\mathrm{E}}\right) x_{\mathrm{E}} \cos 2 \phi+\mathcal{O}\left(\epsilon^{2}\right), \\
\frac{\mathrm{d} f_{0}}{\mathrm{~d} \phi} & =\frac{a_{2}-a_{1}}{2} x_{\mathrm{E}}^{2} \sin 2 \phi+\mathcal{O}\left(\epsilon^{2}\right), \\
\frac{\mathrm{d}^{2} f_{0}}{\mathrm{~d} \phi^{2}} & =\left(a_{2}-a_{1}\right) x_{\mathrm{E}}^{2} \cos 2 \phi+\mathcal{O}\left(\epsilon^{2}\right) .
\end{aligned}
$$

From equation (20) and the expressions above, we have

$x_{\mathrm{t}}(\phi)=x_{\mathrm{E}}\left[1+\frac{a_{2}-a_{1}}{2}\left(\frac{2-\kappa\left(x_{\mathrm{E}}\right)}{\kappa_{2}}\right) \cos 2 \phi\right]$,

and inserting this into equation (27) we get

$\tilde{\sigma}_{R_{\mathrm{th}}}=2 \pi x_{\mathrm{E}}^{2} \frac{R_{\mathrm{th}}^{2}+1}{\left(R_{\mathrm{th}}^{2}-1\right)^{2}}\left[\kappa_{2}^{2}+\frac{1}{8}\left(1+\frac{\kappa_{2}}{2}\right)^{2}\left(a_{2}-a_{1}\right)^{2}\right]$.

Thus, for small ellipticities, the deviation with respect to the axially symmetric case is quadratic.

Instead of using $a_{2}$ and $a_{1}$ it is more intuitive to express the results in terms of the ellipticity of the potential. Several parameterizations have been used to define the ellipticity in this context. From now on, we adopt the convention (Blandford \& Kochanek 1987; Golse \& Kneib 2002; Dúmet-Montoya et al. 2012)

$a_{1}=1-\varepsilon, \quad a_{2}=1+\varepsilon$

where $\varepsilon$ is the potential ellipticity parameter. The connection to the ellipticity of the mass distribution $\varepsilon_{\Sigma}$ depends on the model. For the SIEP $\varepsilon_{\Sigma}=3 \varepsilon$ to first order in $\varepsilon$ (Kassiola \& Kovner 1993). For the PNFW model this relation depends on $\kappa_{s}$ and expressions for $\varepsilon_{\Sigma}\left(\varepsilon, \kappa_{s}\right)$ are provided in Dúmet-Montoya et al. (2012).

Fig. 1 shows the comparison for caustics and critical curves between the PA and the exact solution for the PNFW model for different values of $\kappa_{s}$ and $\varepsilon$.

\subsection{Singular isothermal elliptic potential}

One of the simplest and most often used lens models is given by the SIEP. For this model, using expressions (6) in equation (30), the perturbative functions are

$$
\begin{aligned}
f_{1} & =\Delta_{\phi}-1, \\
\frac{\mathrm{d} f_{0}}{\mathrm{~d} \phi} & =\left(a_{2}-a_{1}\right) \frac{\sin 2 \phi}{2 \Delta_{\phi}}, \\
\frac{\mathrm{d}^{2} f_{0}}{\mathrm{~d} \phi^{2}} & =a_{1} a_{2} \Delta_{\phi}^{-3}-\Delta_{\phi},
\end{aligned}
$$

where $\Delta_{\phi}$ is given in equation (10). When substituted into equations (16) the expressions above lead to

$y_{1}=x\left(1-\frac{a_{1}}{\tilde{x}}\right) \cos \phi$ and $y_{2}=x\left(1-\frac{a_{2}}{\tilde{x}}\right) \sin \phi$,

which are the components of the lens equation of this model without any approximation. Hence, the solution of the PA is exact in the case of lensing by the SIEP model.

The same conclusion does not hold for the PNFW model. We will thus investigate the domain of validity for this model in the next section. 

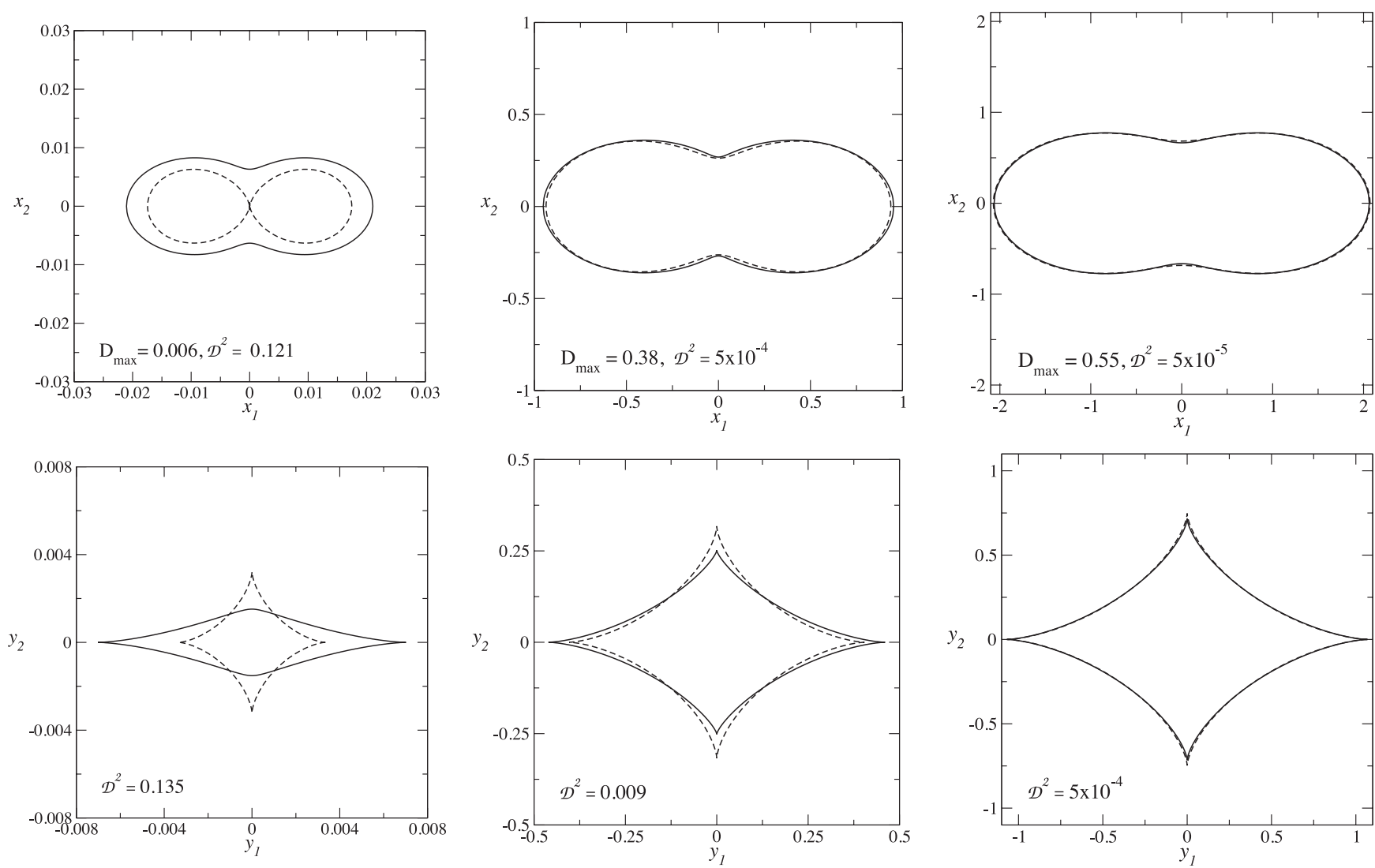

Figure 1. Critical curves (top panels) and caustics (bottom panels) obtained with the PA (dashed lines) and the exact solution (solid lines) for the PNFW model: $\kappa_{s}=0.1$ and $\varepsilon=0.2$ (left), $\kappa_{s}=0.5$ and $\varepsilon=0.32$ (middle), and $\kappa_{s}=1.0$ and $\varepsilon=0.35$ (right). The values of $\varepsilon$ in the middle and right-hand panels were chosen by imposing $\mathcal{D}^{2}=5 \times 10^{-4}$ for critical curves and caustics, respectively (see Section 4 ).

\section{LIMITS OF VALIDITY OF THE PERTURBATIVE APPROACH FOR THE PNFW MODEL}

Previous attempts to quantify the differences between exact and perturbative solutions were carried out in the literature. Alard (2007) proposed a method based on the relative importance of the thirdorder term in the Taylor series of the gravitational potential. Habara \& Yamamoto (2011) performed a qualitative analysis of a particular arc configuration, varying some of the system parameters and establishing criteria based on the position and multiplicity of the images. However, they did not define a metric to compare the solutions nor carry on the analysis for more general configurations.

Investigating the domain of validity of the PA with finite sources would require a large parameter space to be probed, including the lens and source parameters and their relative positions. On the other hand, as a starting point, we may look at quantities that are dependent only on the lens, such as the tangential caustic and critical curve and the deformation cross-section (the latter will depend also on the choice of $R_{\mathrm{th}}$ ). Besides reducing the parameter space - for example, for $\varepsilon$ and $\kappa_{s}$ in the PNFW case - it is simpler to define metrics to quantify the deviation of the perturbed solution from the exact one. We expect that the constraints on the domain of validity determined from the quantities above can be connected to those arising from the images of finite sources. Thus, exploring the simplest case before may provide guidance to the determination of the domain of validity of the method finite sources in the future. Setting a domain of validity from the lens model alone may provide a rapid method to adjudicate validity of the perturbative method a priori, just from the lensing potential, without the need of obtaining images of the sources.

In this section, we shall attempt to quantify the deviation of critical curves and caustics using a figure of merit akin to the one proposed in Dúmet-Montoya et al. (2012). We will then compare the deformation cross-sections and, finally, combine the results to obtain limits that define a region in the parameter space of PNFW models where the PA can be used to accurately obtain local properties of a given lens system.

\subsection{Limits for critical curves and caustics}

To quantify the deviation of the solution of the PA from the exact one for critical curves and caustics we use a figure of merit defined as the mean weighted squared fractional radial difference between the curves, i.e. ${ }^{3}$

$\mathcal{D}^{2} \equiv \frac{\sum_{i=1}^{N} w_{i}\left[x_{\mathrm{ES}}\left(\phi_{i}\right)-x_{\mathrm{PA}}\left(\phi_{i}\right)\right]^{2}}{\sum_{i=1}^{N} w_{i} x_{\mathrm{ES}}^{2}\left(\phi_{i}\right)}$,

where $x_{\mathrm{ES}}\left(\phi_{i}\right)$ and $x_{\mathrm{PA}}\left(\phi_{i}\right)$ are the radial coordinates of the tangential curves (either critical curves or caustics) obtained from the exact solution and with the PA, respectively. These are computed on a discrete set of $N$ points defined by the polar angle $\phi_{i}$. Further,

\footnotetext{
${ }^{3}$ Expression (37) is formally equal to the one proposed in Dúmet-Montoya et al. (2012), where it was used to compare an isocontour of $\kappa$ to an ellipse. Here, the same expression is used to compare two solutions for caustics or critical curves.
} 

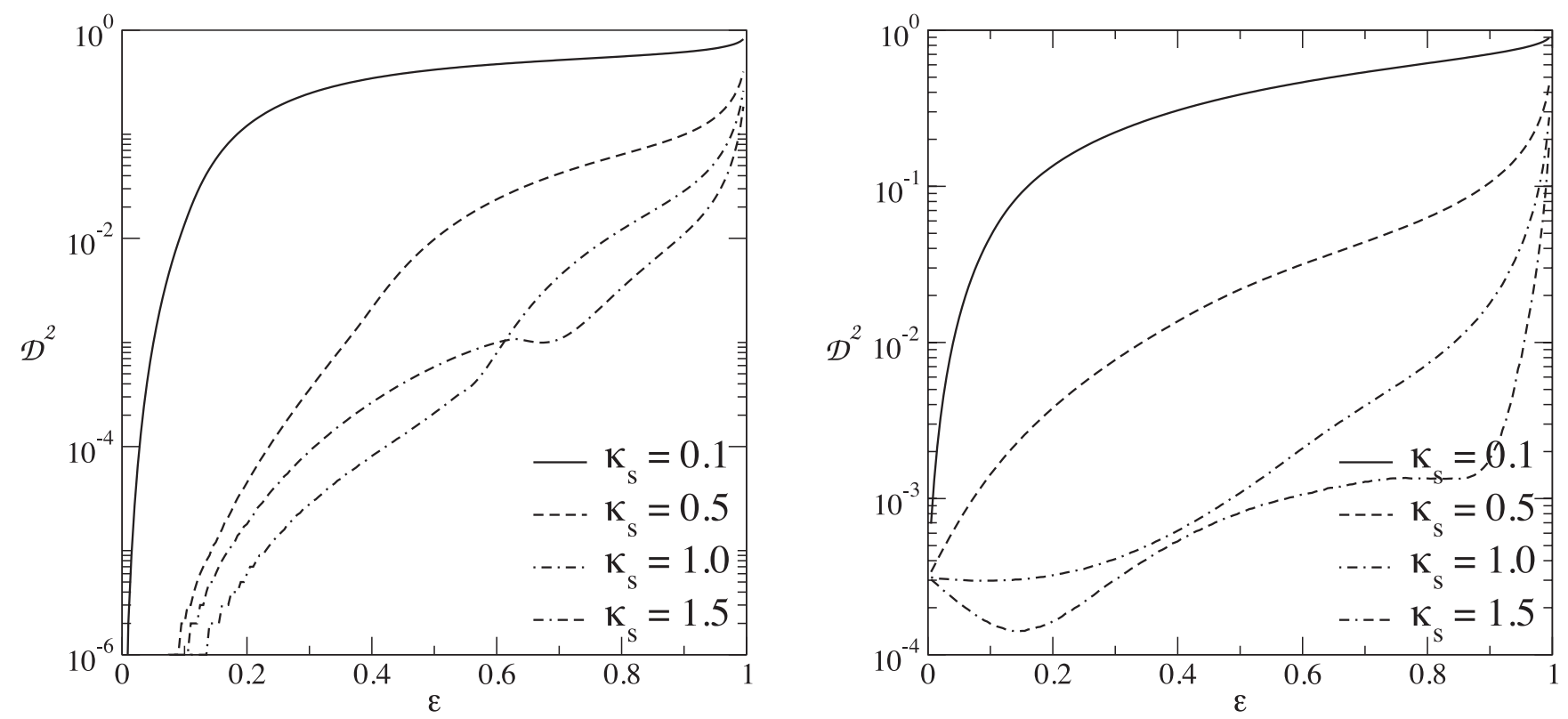

Figure 2. Mean weighted squared radial fractional difference $\mathcal{D}^{2}$ as a function of the ellipticity parameter for the PNFW model for various values of the characteristic convergence $\kappa_{s}$. Left-hand panel: for critical curves. Right-hand panel: for caustics.

$w_{i} \equiv \phi_{i}-\phi_{i-1}$ is a weight to account for a possible non-uniform distribution of points in $\phi$.

Choosing a cut-off value for $\mathcal{D}^{2}$, we can define a range in $\varepsilon$ for which the curves obtained with both the exact and perturbative solutions will be similar enough to each other. The cut-off value is then chosen by visually comparing the exact and perturbative solutions for the critical curves and caustics associated with several values of $\mathcal{D}^{2}$, for combinations of the PNFW lens parameters.

Before presenting the results, we should stress a technical point. In the particular case of caustics, calculating the two functions in the same polar angle becomes a non-trivial issue. This is because in general, the source plane points $\left(y_{1 t}, y_{2 t}\right)$ are not equally distributed in angle, as they are obtained by scanning angular values in the lens plane which map non-linearly to angular values in the source plane. Thus in general, a source plane angle does not correspond to the same lens plane angle. Yet, to compute $\mathcal{D}^{2}$ for the caustics, it is necessary that both $x_{\mathrm{ES}}$ and $x_{\mathrm{PA}}$ be calculated at the same polar angle position in the source plane. Thus, to enforce this last point, we first determine the polar angle corresponding to each point $\left(y_{1 \mathrm{t}}, y_{2 \mathrm{t}}\right)$ obtained with the exact solution, i.e.

$\phi_{\mathrm{S}}=\arctan \left(\frac{y_{2 \mathrm{t}}}{y_{1 \mathrm{t}}}\right)$

and obtain the corresponding radial coordinate $x_{\mathrm{ES}}=y_{\mathrm{t}}\left(\phi_{\mathrm{S}}\right)=$ $\sqrt{y_{1 t}^{2}+y_{2 t}^{2}}$. In the same way, we compute the polar angle of the tangential caustic obtained with the PA (which we denote by $\phi_{\mathrm{S}}^{\mathrm{PA}}$ ), i.e.

$\phi_{\mathrm{S}}^{\mathrm{PA}}=\arctan \left(\frac{y_{2 \mathrm{t}}\left(\phi_{L}\right)}{y_{1 \mathrm{t}}\left(\phi_{L}\right)}\right)$,

where $y_{1 \mathrm{t}}$ and $y_{2 \mathrm{t}}$ are given in equation (21) and $y_{\mathrm{t}}^{\mathrm{PA}}=\sqrt{y_{1 \mathrm{t}}^{2}+y_{2 \mathrm{t}}^{2}}$. We then vary the angle $\phi_{L}$ (only the interval $0 \leq \phi_{L} \leq \pi / 2$ is needed, for symmetry reasons) such that for each radial position $y_{\mathrm{t}}$, the angles $\phi_{\mathrm{S}}$ and $\phi_{\mathrm{S}}^{\mathrm{PA}}$ are chosen to have the same value at step $i$. Finally, having determined $\left(y_{\mathrm{t}}, \phi_{\mathrm{S}}\right)$ for the exact solution and $\left(y_{\mathrm{t}}^{\mathrm{PA}}, \phi_{\mathrm{S}}^{\mathrm{PA}}\right)$, we proceed to compute $\mathcal{D}^{2}$ as in equation (37).
Fig. 2 shows $\mathcal{D}^{2}$ as a function of $\varepsilon$ for some values of ${ }^{4} \kappa_{s}$. In the left-hand panel, the results for critical curves are shown. Since the perturbation increases with $\varepsilon, \mathcal{D}^{2}$ also increases with $\varepsilon$, as we might expect. In addition, $\mathcal{D}^{2}$ decreases as $\kappa_{s}$ increases, at least for $\kappa_{s}<$ 1.0. In the right-hand panel, we show the results for caustics. The behaviour of $\mathcal{D}^{2}$ is qualitatively similar to that of critical curves, except for at the highest $\kappa_{s}$, where the behaviours are reversed. However, the values of $\mathcal{D}^{2}$ computed for caustics are higher than the corresponding ones for critical curves, for a given $\left(\kappa_{s}, \varepsilon\right)$. This means that imposing cut-off values of $\mathcal{D}^{2}$ for matching caustics, we will match the corresponding critical curves automatically. We found by visual inspection that for $\mathcal{D}^{2} \leq 5 \times 10^{-4}$ there is a very good match for the caustic curves. In Fig. 1, we show the values of $\mathcal{D}^{2}$ calculated for each example, demonstrating visually the validity of this diagnostic measure. In particular, we have checked that cutoff values of $\mathcal{D}^{2}$ higher but close to our chosen limit of $5 \times 10^{-4}$ are not suited for matching caustic curves well.

To estimate the validity of the PA, Alard (2007) introduced the parameter $D \equiv 3\left|C_{3}\right|\left(\delta x_{\mathrm{arc}}\right)^{2}$, where $\delta x_{\text {arc }}$ corresponds to the difference between the arc contours obtained in the PA and the Einstein radius, and $C_{3}$ is the third-order term in the Taylor expansion of the gravitational potential (see equation 13). In order for the PA to be accurate, $D$ should be small. For models based on the SIS profile, this condition is always true, since $C_{3}=0$ (which is consistent with the fact that the perturbative method is exact in this case). For other pseudo-elliptical models, usually $C_{3} \neq 0$.

Here, we adapt the definition of $D$ to be used for critical curves, such that $\delta x$ is now the radial deviation of these curves with respect to $x_{E}$. We associate a unique value of $D$ to the tangential critical curve, using its maximum value over this curve, which corresponds to

$D_{\max }=3\left|C_{3}\right| \max \left\{\left(x_{\mathrm{t}}(\phi)-x_{\mathrm{E}}\right)^{2}\right\}$,

\footnotetext{
${ }^{4}$ Throughout this work, following Dúmet-Montoya et al. (2012), we will consider the range $\kappa_{s} \leq 1.5$.
} 


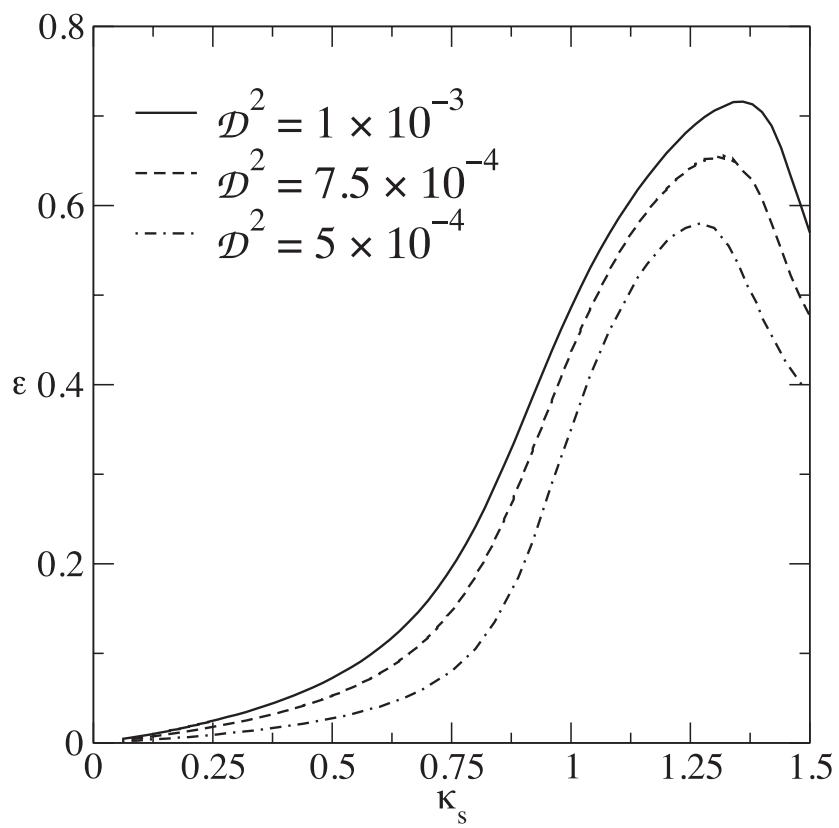

Figure 3. Maximum values of $\varepsilon$ obtained from some cut-off values of $\mathcal{D}^{2}$ on caustics for the PNFW model.

where $x_{\mathrm{t}}$ is given in equation (20) and $0 \leq \phi \leq 2 \pi$. Following Alard's criterion (i.e. $D_{\max } \ll 1$ ), it would be expected that the critical curves and caustics obtained with the PA would be close to the ones obtained in the exact case when both $\varepsilon$ and $\kappa_{s}$ are small. We compute $D_{\max }$ for the curves shown in Fig. 1, obtaining $D_{\max }=$ $0.006,0.38$ and 0.55 from left- to right-hand panels. In contrast to expectations, when $D_{\max }$ increases, the curves obtained with the PA become more similar to the exact solutions. Therefore, the criterion $D_{\max } \ll 1$ does not reflect the validity of the PA for these cases. Moreover, $D_{\max }$ is not scale invariant (i.e. $D_{\max } \propto r_{\mathrm{s}}^{2}$, where $r_{\mathrm{s}}$ is the length-scale of the PNFW model). These considerations show that this measure is not well suited to assess the limit of validity of the method for caustics and critical curves. This result emphasizes the relevance of our definition of $\mathcal{D}^{2}$ as a measure for the validity of the PA for critical curves and caustics.

For the application of our criterion, we define $\varepsilon_{\max }^{\mathrm{PA}}$, for a given $\kappa_{s}$, as the ellipticity threshold giving $\mathcal{D}^{2}=5 \times 10^{-4}$. This will be used as a measure of the limit of applicability for the PA for critical curves and caustics. Fig. 3 shows the maximum values of $\varepsilon$ as a function of $\kappa_{s}$ for the PNFW model, for some cut-off values of $\mathcal{D}^{2}$. The $\varepsilon_{\max }^{\mathrm{PA}}\left(\kappa_{s}\right)$ function shown in this figure is well fitted by a Padé approximant of the form

$\varepsilon_{\max }^{\mathrm{PA}}=\frac{\sum_{n=0}^{4} a_{n}\left(\kappa_{s}\right)^{n}}{\sum_{m=0}^{2} b_{m}\left(\kappa_{s}\right)^{m}}$,

with $a_{n}=\{-0.018,0.235,-0.415,0.565,-0.264\}$ and $b_{n}=$ $\{2.243,-3.709,1.725\}$.

\subsection{Comparison between deformation cross-sections}

In this section, we compare the exact and perturbative solutions for the deformation cross-section in order to establish limits of validity for the approximation of this quantity. We then contrast these limits to those obtained for caustics and critical curves as done in Section 4.1 (i.e. by imposing $\varepsilon<\varepsilon_{\max }^{\mathrm{PA}}$ for each $\kappa_{s}$ ). If within this regime the PA and the exact solution of the deformation cross-section do not agree well, this can impose additional limits to the applicability of the PA.

To quantify the difference between the deformation crosssections, we compute their relative difference

$\frac{\Delta \tilde{\sigma}_{R_{\mathrm{th}}}}{\tilde{\sigma}_{R_{\mathrm{th}}}}=\left|\frac{\tilde{\sigma}_{\mathrm{ES}, \mathrm{R}_{\mathrm{th}}}-\tilde{\sigma}_{\mathrm{PA}, \mathrm{R}_{\mathrm{th}}}}{\tilde{\sigma}_{\mathrm{ES}, \mathrm{R}_{\mathrm{th}}}}\right|$,

where the subscripts ES and PA refer to the exact and perturbative calculations, respectively.

In Fig. 4 , we show $\Delta \tilde{\sigma}_{10} / \tilde{\sigma}_{10}$ as a function of $\kappa_{s}$ for some values of $\varepsilon$. In the left-hand panel, we compare the exact solution with the expansion for low ellipticities in the PA, equation (33), while in the right-hand panel we compare with the general expression, equation (27). ${ }^{5}$ The perturbative calculation for the axially symmetric NFW model $(\varepsilon=0$, equation 28$)$ is a good approximation in this case, since $\Delta \tilde{\sigma}_{10} / \tilde{\sigma}_{10}<10$ per cent for the entire allowed range of $\kappa_{s}$. For values of $\varepsilon<0.1$ the PA is a good approximation only for $\kappa_{s} \gtrsim$ 0.5 . As $\varepsilon$ increases, the difference is larger at smaller values of $\kappa_{s}$. However, the perturbative calculation is accurate to within about 10 per cent for $\kappa_{s} \gtrsim 0.7$ up to $\varepsilon=0.3$ (see the right-hand panel of Fig. 4).

Additionally, we computed $\Delta \tilde{\sigma}_{\text {th }} / \tilde{\sigma}_{\text {th }}$ as a function of the threshold $R_{\text {th }}$. We find that $\Delta \tilde{\sigma}_{\text {th }} / \tilde{\sigma}_{\text {th }}$ can exceed 50 percent at values of $R_{\mathrm{th}} \lesssim 2.5$, since for these values of $R_{\mathrm{th}}$, the constant distortion curves are far from the tangential critical curves, meaning that the premises of the PA do not apply. However, as $R_{\text {th }}$ increases, the relative deviations among the deformation cross-sections decrease. In particular, we found that for $\kappa_{s} \gtrsim 0.9$ and $R_{\mathrm{th}}>7.5$, these relative deviations do not depend on $R_{\text {th }}$.

In Fig. 5, we show isocontours of $\Delta \tilde{\sigma}_{10} / \tilde{\sigma}_{10}$, for the exact and perturbative calculations, in the parameter space $\kappa_{s}-\varepsilon$ together with the curve $\varepsilon_{\max }^{\mathrm{PA}}\left(\kappa_{s}\right)$. We see that the constraints imposed by $\Delta \tilde{\sigma}_{10} / \tilde{\sigma}_{10}$ and $\varepsilon_{\max }^{\mathrm{PA}}$ are complementary, meaning that for $\kappa_{s} \lesssim 1.0$ the constraint obtained with caustics and critical curves is the strongest, while the opposite is true for $\kappa_{s}>1.0$ if we impose that the maximum fractional deviation for the cross-section is 10 per cent.

We may then combine the constraints to define a region limited approximately by the curves

$\varepsilon=\left\{\begin{array}{cc}\varepsilon_{\max }^{\mathrm{PA}}\left(\kappa_{s}\right), & \kappa_{s} \lesssim 1.0, \\ 0.33, & \kappa_{s}>1.0 .\end{array}\right.$

Within this region the PA can replace the exact computation of critical curves, caustics and deformation cross-section with high accuracy.

\section{CONCLUDING REMARKS}

The PA (Alard 2007, 2008, 2009, 2010) provides analytical solutions for gravitational arcs by solving the lens equation linearized around the Einstein ring solution. This method has a wide range of potential applications, from the inverse problem in strong lensing to fast arc simulations. This technique goes beyond other analytical approximations in the literature in which it may be used for generic lens models (including mass distributions arising from $N$-body simulations) and for finite sources.

\footnotetext{
${ }^{5}$ It should be noted that, due to the quadratic scaling with respect to $\epsilon$ of the deviation from the axially symmetric case, equation (33) is an excellent approximation for equation (27) for low ellipticities. Thus, the left-hand panel of Fig. 4 would remain essentially unchanged if we used equation (27) instead of equation (33) there.
} 

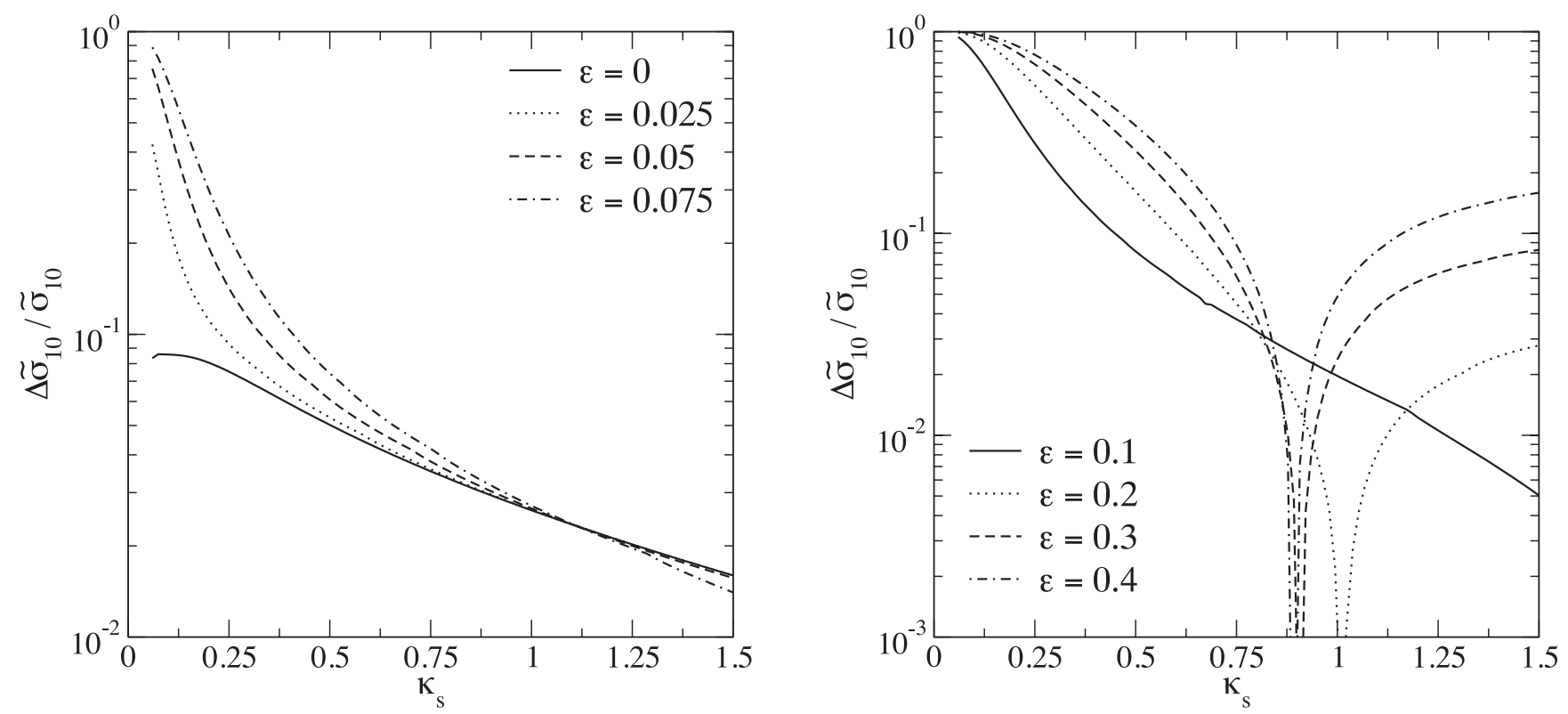

Figure 4. Relative deviation among deformation cross-sections for the PNFW model as a function of $\kappa_{s}$ for some values of $\varepsilon$. Left-hand panel: $\Delta \tilde{\sigma}_{10} / \tilde{\sigma}_{10}$ between exact solution and equation (33). Right-hand panel: $\Delta \tilde{\sigma}_{10} / \tilde{\sigma}_{10}$ between exact solution and equation (27).

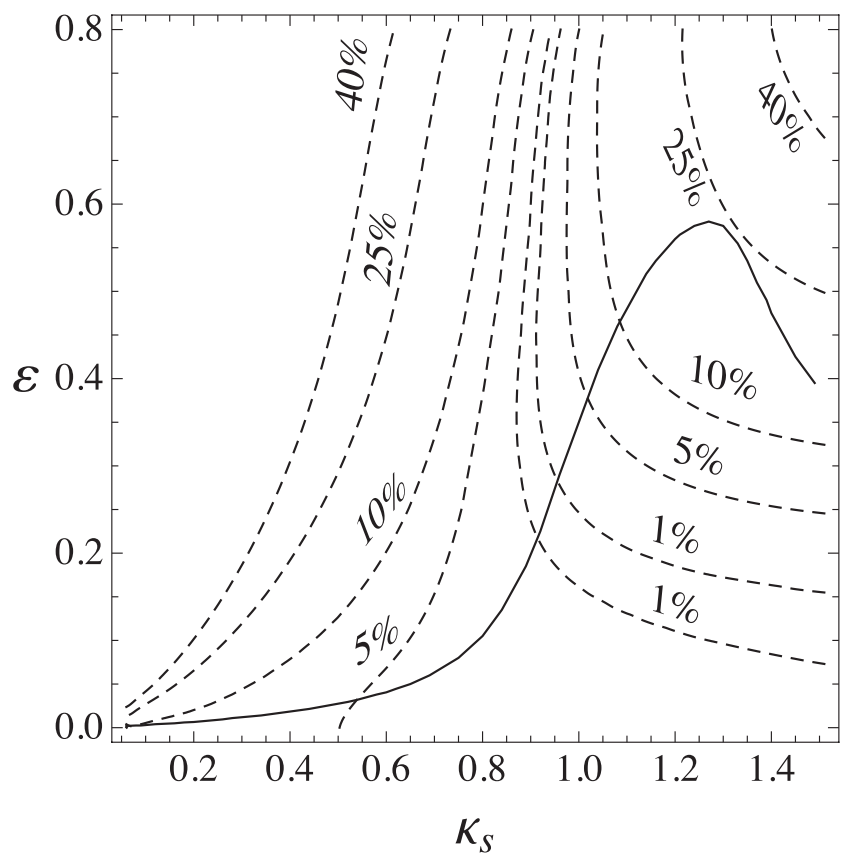

Figure 5. Comparison between deformation cross-sections for the PNFW model space parameter. Contours of constant $\Delta \tilde{\sigma}_{10} / \tilde{\sigma}_{10}$. The solid line shows the $\varepsilon_{\max }^{\mathrm{PA}}\left(\kappa_{s}\right)$ curve.

A key aspect for practical applications of the method that has not been systematically addressed before is the determination of its limit of validity. Motivated by this issue, in this paper we aimed to determine the accuracy of the PA for caustics and critical curves, and for the deformation arc cross-section. Although these quantities do not involve arcs (i.e. the lensing of finite sources), they allow one to obtain limits on the accuracy of the linearized mapping from the PA. Also, the parameter space to be probed is significantly decreased, since these quantities depend basically on the lens properties and not on the source ones.
We have considered a restricted set of lens models, more specifically those with elliptical lens potentials, and in particular the PNFW and SIEP models, which are nevertheless widely used in strong lensing applications, specially for the inverse modelling. Whenever possible, we sought to derive analytic expressions for the quantities involved in the calculations, many of which are new. Some are valid for the PA in general, others apply to pseudo-elliptic lens models. The main results of the paper are summarized below.

We obtained analytic expressions for the constant distortion curves in the PA (equations 23 and 24), which, in the lens plane, are found to be self-similar to the tangential critical curve. We derived an analytic formula for the deformation cross-section (equation 27), which reproduces the scaling of the arc cross-section with $R_{\text {th }}$ obtained numerically in previous works. For axially symmetric models, the cross-section is obtained in closed form (equation 28).

We have obtained simple analytic expressions for the perturbative functions for pseudo-elliptical models, which are valid for any choice of the ellipticity parametrization (equation 30). These expressions generalize those given in Alard $(2007,2008)$ and in Habara \& Yamamoto (2011).

We derive approximate solutions to the tangential critical curve (equation 32) and for the deformation cross-section (equation 33) for low ellipticities in pseudo-elliptical models. We show that the deviation of the cross-section with respect to the axially symmetric case is quadratic in the ellipticity.

We have considered the SIEP and the PNFW models to represent lenses at galaxy and galaxy cluster mass scales. We have shown that the PA provides the exact solution for the SIEP model. For the PNFW model, we compared the critical curves and caustics obtained with this approach with those obtained with the exact solution for a wide range of values of $\kappa_{s}$ and $\varepsilon$.

We show that the criterion $D_{\max } \ll 1$ proposed by Alard (2007) extended to be applied to the tangential critical curve (equation 38 ) is not adequate to set a limit of validity for these cases. To this end, we use a figure of merit, $\mathcal{D}^{2}$ (equation 37 ), to quantify the deviation of the PA from the exact solution for caustics and critical curves. We verify that $\mathcal{D}^{2}$ provides a quantitative description of the 
deviation among both solutions. In particular, $\mathcal{D}^{2}$ decreases with $\kappa_{s}$ (as can be drawn from Fig. 1) and increases with $\varepsilon$ (as expected from the increasing of the perturbation to the lensing potential with $\varepsilon$ ). Since the deviation between the exact and perturbative solutions for caustics is higher than the deviation for critical curves, it is sufficient to set a limit on $\mathcal{D}^{2}$ for caustics to ensure a small deviation for critical curves.

By setting a threshold on $\mathcal{D}^{2}$ computed at caustics, a maximum value of $\varepsilon$ is determined for each $\kappa_{s}$, such that a good matching for caustics and also for critical curves is ensured. We determine these maximum values $\varepsilon_{\max }^{\mathrm{PA}}\left(\kappa_{s}\right)$ by choosing $\mathcal{D}^{2}=5 \times 10^{-4}$. This defines a domain of applicability of the PA for the PNFW model in the range of $\kappa_{s}$ being considered. We provide a fitting function for $\varepsilon_{\max }^{\mathrm{PA}}\left(\kappa_{s}\right)$ (equation 39). For $\kappa_{s} \lesssim 0.8$, the PA is limited to $\varepsilon \lesssim 0.1$. However, for $\kappa_{s}>1.0$ it is possible to use this approach even up to $\varepsilon=0.4$ for these cases.

Another limit on the PFNW model parameters is obtained from the comparison of the deformation cross-section for both exact and perturbative calculations. The fractional deviation is less than 10 percent (Fig. 5) for $\kappa_{s} \gtrsim 0.7$ and $\varepsilon \lesssim 0.3$ (corresponding to $\left.\epsilon_{\Sigma} \lesssim 0.55\right)$.

We may use these results to set further constraints on the ellipticity parameter of the PNFW model, by requiring an agreement with the exact $\tilde{\sigma}_{R_{\mathrm{th}}}$, besides the condition $\varepsilon<\varepsilon_{\max }^{\mathrm{PA}}\left(\kappa_{s}\right)$. This ensures that caustics, critical curves and the local mapping are well reproduced by the PA for the PNFW model. The combined restriction, imposing the matching for caustics and an agreement to about 10 per cent for deformation cross-sections, is given in equation (41).

In this paper, we provided a first systematic attempt to set limits on the domain of applicability of the PA for strong lensing in terms of the parameters of a given lens model, more specifically for the PNFW model. The limits are imposed so that the caustics, critical curves and deformation cross-section match the exact solutions with a given accuracy. Although these quantities are useful for strong lensing applications, it is important to determine a domain of validity for arcs/finite sources. For example, Habara \& Yamamoto (2011) investigated the domain of validity of the PA for extended circular sources. It is argued that PA can be used for sources with radius $\lesssim 0.2 x_{\mathrm{E}}$ up to $\varepsilon \simeq 0.3$. This result should be extended for generic configurations probing the space of the source and lens parameters and their relative position. We expect that the limits obtained here can be connected to the domain of validity for arcs providing guidance to the exploration of this wider parameter space. The systematic application to arcs and connection to the current results is left for a subsequent work. It is also important to check whether the criterion established here for the $\mathcal{D}^{2}$ threshold can be applied to other lens models, so that we have an a priori criterion for the domain of validity of the PA regardless of the specific model.

The usefulness of the PA justifies the search for a determination of its accuracy and limit of applicability. Once this is established we will be able to safely use this promising technique in a number of applications, within its domain of validity.

\section{ACKNOWLEDGEMENTS}

HSDM is funded by the Brazilian agencies FAPERJ (E-26/101.784/2010), CNPq (PDJ/162989/2011-3) and the PCI/MCTI programme at CBPF (301.860/2011-4). GBC is funded by $\mathrm{CNPq}$ and CAPES. BM is supported by FAPERJ. MM is partially supported by CNPq (grant 312876/2009-2) and FAPERJ (grant E-26/110.516/2012). MSSG acknowledges the hospitality of the
Centro Brasileiro de Pesquisas Físicas (CBPF), where part of this work was performed and the PCI/MCTI programme (170.524/2006$0)$. We also thank the support of the Laboratório Interinstitucional de e-Astronomia (LIneA) operated jointly by CBPF, the Laboratório Nacional de Computação Científica (LNCC) and the Observatório Nacional (ON), and funded by the Ministry of Science, Technology and Innovation (MCTI). MM acknowledges C. Alard for useful discussions regarding the PA for arcs. We thank Marcos Lima for useful discussions.

\section{REFERENCES}

Abbott T. et al., 2005, preprint (astro-ph/0510346)

Alard C., 2007, MNRAS, 382, L58

Alard C., 2008, MNRAS, 388, 375

Alard C., 2009, A\&A, 506, 609

Alard C., 2010, A\&A, 513, A39

Annis J. et al., 2005, preprint (astro-ph/0510195)

Bartelmann M., 1996, A\&A, 313, 697

Bartelmann M., Weiss A., 1994, A\&A, 287, 1

Bartelmann M., Steinmetz M., Weiss A., 1995, A\&A, 297, 1

Bartelmann M., Huss A., Colberg J., Jenkins A., Pearce F., 1998, A\&A, 330,1

Bartelmann M., Meneghetti M., Perrotta F., Baccigalupi C., Moscardini L., 2003, A\&A, 409, 449

Bayliss M. B., 2012, ApJ, 744, 156

Belokurov V., Evans N. W., Hewett P. C., Moiseev A., McMahon R. G., Sanchez S. F., King L. J., 2009, MNRAS, 392, 104

Blandford R. D., Kochanek C. S., 1987, ApJ, 321, 658

Boldrin M., Giocoli C., Meneghetti M., Moscardini L., 2012, MNRAS, 427, 3134

Cabanac R. A. et al., 2007, A\&A, 461, 813

Comerford J. M., Meneghetti M., Bartelmann M., Schirmer M., 2006, ApJ, 642,39

Dobler G., Keeton C. R., 2006, MNRAS, 365, 1243

Dúmet-Montoya H. S., 2011, PhD thesis, CBPF

Dúmet-Montoya H. S., Caminha G. B., Makler M., 2012, A\&A, 544, 83

Estrada J. et al., 2007, ApJ, 660, 1176

Fedeli C., Meneghetti M., Bartelmann M., Dolag K., Moscardini L., 2006, A\&A, 447, 419

Furlanetto C. et al., 2013a, MNRAS, 432, 73

Furlanetto C. et al., 2013b, A\&A, 549, A80

Gilbank D. G., Gladders M. D., Yee H. K. C., Hsieh B. C., 2011, AJ, 141, 94

Gladders M. D., Hoekstra H., Yee H. K. C., Hall P. B., Barrientos L. F., 2003, ApJ, 593, 48

Golse G., Kneib J.-P., 2002, A\&A, 390, 821

Golse G., Kneib J.-P., Soucail G., 2002, A\&A, 387, 788

Grossman S. A., Saha P., 1994, ApJ, 431, 74

Habara Y., Yamamoto K., 2011, Int. J. Mod. Phys. D, 20, 371

Hamana T., Futamase T., 1997, MNRAS, 286, L7

Hattori M., Watanabe K., Yamashita K., 1997, A\&A, 319, 764

Hennawi J. F. et al., 2008, AJ, 135, 664

Horesh A., Maoz D., Hilbert S., Bartelmann M., 2011, MNRAS, 418, 54

Horesh A., Maoz D., Ebeling H., Seidel G., Bartelmann M., 2012, MNRAS, 406, 1318

Jullo E., Kneib J.-P., Limousin M., Elíasdóttir Á., Marshall P. J., Verdugo T., 2007, New J. Phys., 9, 447

Jullo E., Natarajan P., Kneib J.-P., D’Aloisio A., Limousin M., Richard J., Schimd C., 2010, Sci, 329, 924

Kassiola A., Kovner I., 1993, ApJ, 417, 450

Kausch W., Schindler S., Erben T., Wambsganss J., Schwope A., 2010, A\&A, 513, A8

Keeton C. R., 2001a, ApJ, 562, 160

Keeton C. R., 2001b, preprint (astro-ph/0102340)

Kneib J.-P., 2001, preprint (astro-ph/0112123)

Kneib J.-P., Mellier Y., Fort B., Mathez G., 1993, A\&A, 273, 367 
Kneib J.-P., Van Waerbeke L., Makler M., Leauthaud A., 2010, CFHT programs 10BF023, 10BC022, 10BB009

Kovner I., 1989, ApJ, 337, 621

Kubo J. M. et al., 2010, ApJ, 724, L137

Luppino G. A., Gioia I. M., Hammer F., Le Fèvre O., Annis J. A., 1999, A\&AS, 136, 117

Meneghetti M., Yoshida N., Bartelmann M., Moscardini L., Springel V., Tormen G., White S. D. M., 2001, MNRAS, 325, 435

Meneghetti M., Bartelmann M., Moscardini L., 2003, MNRAS, 340, 105

Meneghetti M., Bartelmann M., Jenkins A., Frenk C., 2007, MNRAS, 381, 171

Meneghetti M. et al., 2008, A\&A, 482, 403

Miralda-Escudé J., 1993a, ApJ, 403, 497

Miralda-Escudé J., 1993b, ApJ, 403, 509

Mollerach S., Roulet E., 2002, Gravitational Lensing and Microlensing. World Scientific Press, Singapore

More A., Cabanac R., More S., Alard C., Limousin M., Kneib J.-P., Gavazzi R., Motta V., 2012, ApJ, 749, 38

Navarro J. F., Frenk C. S., White S. D. M., 1996, ApJ, 462, 563

Navarro J. F., Frenk C. S., White S. D. M., 1997, ApJ, 490, 493

Oguri M., 2002, ApJ, 573, 51

Oguri M., 2010, PASJ, 62, 1017

Oguri M., Taruya A., Suto Y., 2001, ApJ, 559, 572
Oguri M., Lee J., Suto Y., 2003, ApJ, 599, 7 [erratum: ibid, 608, 1175 (2004)]

Peirani S., Alard C., Pichon C., Gavazzi R., Aubert D., 2008, MNRAS, 390, 945

Petters A. O., Levine H., Wambsganss J., 2001, Singularity Theory and Gravitational Lensing. Birkhäuser, Boston

Rozo E., Nagai D., Keeton C., Kravtsov A., 2008, ApJ, 687, 22

Sand D. J., Treu T., Ellis R. S., Smith G. P., 2005, ApJ, 627, 32

Schneider P., Elhers J., Falco E. E., 1992, Gravitational Lenses. SpringerVerlag, Berlin

Smith G. P., Kneib J.-P., Smail I., Mazzotta P., Ebeling H., Czoske O., 2005, MNRAS, 359, 417

Turner E. L., Ostriker J. P., Gott J. R., 1984, ApJ, 284, 1

van de Ven G., Mandelbaum R., Keeton C. R., 2009, MNRAS, 398, 607

Wayth R. B., Webster R. L., 2006, MNRAS, 372, 1187

Wen Z.-L., Han J.-L., Jiang Y.-Y., 2011, Res. Astron. Astrophys., 11, 1185

Werner M. C., An J., Evans N. W., 2008, MNRAS, 391, 668

Wiesner M. P. et al., 2012, ApJ, 761, 1

Wu X. P., Hammer F., 1993, MNRAS, 262, 187

Zaritsky D., Gonzalez A. H., 2003, ApJ, 584, 691

This paper has been typeset from a $\mathrm{T}_{\mathrm{E}} \mathrm{X} / \mathrm{LT}_{\mathrm{E}} \mathrm{X}$ file prepared by the author. 